\title{
CONTENTS BY SUBJECT AREA
}

MAJOR THEMES

Applied Ecology

Behavioral Ecology

Computational Ecology

Ecosystem Ecology

Epidemiology and Epidemic Modeling

Population Ecology

Spatial Ecology

Statistics in Ecology

PHYSIOLOGICAL AND BIOPHYSICAL ECOLOGY

Allometry and Growth

Belowground Processes

Energy Budgets

Functional Traits of Species and Individuals

Hydrodynamics

Integrated Whole Organism Physiology

Plant Competition and Canopy Interactions

Transport in Individuals

POPULATION DYNAMICS

Age Structure

Allee Effects

Apparent Competition

Beverton-Holt Model

Cannibalism

Chaos
Demography

Disease Dynamics

Dispersal, Animal

Dispersal, Plant

Ecosystem Engineers

Facilitation

Food Chains and Food Web Modules

Foraging Behavior

Invasion Biology

Mating Behavior

Metapopulations

Movement: From Individuals to Populations

Nicholson-Bailey Host Parasitoid Model

NPZ Models

Predator-Prey Models

Ricker Model

Single-Species Population Models

SIR Models

Spatial Spread

Species Ranges

Stage Structure

Stochasticity, Demographic

Stochasticity, Environmental

Stress and Species Interactions

Synchrony, Spatial

Two-Species Competition 
EVOLUTIONARY ECOLOGY

Adaptive Behavior and Vigilance

Adaptive Dynamics

Adaptive Landscapes

Coevolution

Cooperation, Evolution of

Dispersal, Evolution of

Evolutionarily Stable Strategies

Evolutionary Computation

Mutation, Selection, and Genetic Drift

Niche Construction

Phenotypic Plasticity

Phylogenetic Reconstruction

Phylogeography

Quantitative Genetics

Sex, Evolution of

COMMUNITY ECOLOGY

Assembly Processes

Bottom-Up Control

Diversity Measures

Food Webs

Metacommunities

Microbial Communities

Neutral Community Ecology

Niche Overlap

Regime Shifts

Resilience and Stability

Storage Effect

Succession

Top-Down Control

\section{ECOSYSTEM ECOLOGY}

Biogeochemistry and Nutrient Cycles

Compartment Models

Continental Scale Patterns

Environmental Heterogeneity and Plants

Forest Simulators

Gas and Energy Fluxes across Landscapes

Geographic Information Systems

Landscape Ecology

Metabolic Theory of Ecology

Ocean Circulation, Dynamics of

Stoichiometry, Ecological
MATHEMATICAL APPROACHES

Bayesian Statistics

Bifurcations

Birth-Death Models

Branching Processes

Cellular Automata

Delay Differential Equations

Difference Equations

Dynamic Programming

Frequentist Statistics

Game Theory

Individual-Based Ecology

Information Criteria in Ecology

Integrodifference Equations

Markov Chains

Matrix Models

Meta-Analysis

Model Fitting

Networks, Ecological

Nondimensionalization

Optimal Control Theory

Ordinary Differential Equations

Pair Approximations

Partial Differential Equations

Phase Plane Analysis

Reaction-Diffusion Models

Spatial Models, Stochastic

Stability Analysis

Stochasticity (Overview)

\section{APPLICATIONS}

Conservation Biology

Discounting in Bioeconomics

Ecological Economics

Ecosystem Services

Ecosystem Valuation

Ecotoxicology

Fisheries Ecology

Gap Analysis and Presence/Absence Models

Harvesting Theory

Marine Reserves and Ecosystem-Based Management

Population Viability Analysis

Reserve Selection and Conservation Prioritization

Restoration Ecology

Urban Ecology 